\title{
Activation of a prophage-encoded tyrosine kinase by a heterologous infecting phage results in a self-inflicted abortive infection
}

David I. Friedman, ${ }^{1 *}$ Cara C. Mozola, ${ }^{1 \dagger}$ Karen Beeri, ${ }^{1 \ddagger}$ Ching-Chung $\mathrm{Ko}^{2}$ and Jared L. Reynolds ${ }^{1 \S}$ ${ }^{1}$ Department of Microbiology and Immunology, University of Michigan, Ann Arbor, MI 48109, USA. ${ }^{2}$ Pittsburgh Bacteriophage Institute, Department of Biological Sciences, University of Pittsburgh, Pittsburgh, PA 15260, USA.

\section{Summary}

Bacteria in their struggle for survival have evolved or acquired defences against attacking phage. However, phage often contribute to this defence through mechanisms in which a prophage protects the bacterial population from attack by another, often unrelated, phage. The 933W prophage, which carries Shiga toxin genes that enhance pathogenicity of enterohaemorrhagic Escherichia coli strain 0157:H7, also carries the stk gene encoding a eukaryotic-like tyrosine kinase that excludes (aborts) infection by phage HK97. This exclusion requires the kinase activity of Stk. Little, if any, protein tyrosine phosphorylation can be detected in a $933 \mathrm{~W}$ lysogen prior to infection with HK97, while extensive Stk-mediated tyrosine phosphorylation is evident following infection. This includes autophosphorylation that stabilizes Stk protein from degradation. Although increased levels of Stk are found following HK97 infection, these higher levels are not necessary or sufficient for exclusion or protein phosphorylation. An HK97 open reading frame, orf41, is necessary for exclusion and Stk kinase activity. We hypothesize that interaction with gp41 stimulates Stk kinase activity. Exclusion of HK97 appears to be specific since other phages tested, $\lambda$, $\phi 80, \mathrm{H}-19 \mathrm{~B}, \lambda$-P22dis and T4rll, were not excluded. Infection of the $933 \mathrm{~W}$ lysogen with a non-excluded phage fails to induce Stk-determined phosphorylation.

Accepted 9 September, 2011. *For correspondence. E-mail davidfri@ umich.edu; Tel. (+1) 734763 3142; Fax (+1) 734764 3562. Present addresses: 'Department of Molecular Microbiology, Washington University, St. Louis, MO 63110-1093, USA; `8252 Regents Rd apt 203, San Diego, CA 92122, USA; §Centers for Disease Control and Prevention, Atlanta, GA 30333, USA.

\section{Introduction}

Bacteria, under constant threat of attack by phage, have evolved or acquired an array of defences against these viral as well as other assailants, e.g. restriction systems (Arber, 1971), CRISPR (Brouns et al., 2008) and toxinantitoxin systems (Fineran et al., 2009). Prophage, through a variety of mechanisms referred to as exclusion also can contribute to the defence of their bacterial hosts (Duckworth et al., 1981; Snyder, 1995; Gottesman, 1998; Chopin et al., 2005). Some exclusion systems block entry of the infecting phage or destroy the infecting DNA, while others abort infection resulting in death of the infected bacterium before infectious phage can be produced, a dead end for the attacking phage. Although the infected bacterium dies, the failure in production of the attacking phage prevents spread to other members of the bacterial population. Hence, the resident prophage, while not protecting its own host bacterium, indirectly protects the remaining members of the population. Death of a fraction of a bacterial population to protect the remaining population has colloquially been referred to as altruistic behaviour (Shub, 1994; Ackermann et al., 2008), a behaviour widely observed in nature (West and Gardner, 2010). Historically, Rex exclusion (Fig. 1A) expressed by $\lambda$ prophage (Howard, 1967; Matz et al., 1982; Parma et al., 1992), which aborts infection of T4rll mutants, gained broad recognition through its use in classic molecular genetic studies (Benzer, 1966).

Coliphage 933W was isolated from the $0157: \mathrm{H} 7$ enterohaemorrhagic Escherichia coli (EHEC) strain EDL933 (O'Brien et al., 1984). The phage genome includes genes encoding Shiga toxin 2 (stx $2 A$ and $B$ ), the virulence factor responsible for the most serious sequelae of EHEC infection (Kaper et al., 2004). 933W is a member of the lambdoid family of phages (Tyler et al., 2005), so named because they have genomes arranged similarly to that of $\lambda$ (Fig. 1A), with most genes shared from a large gene pool (Campbell and Botstein, 1983; Campbell, 1994; Hendrix, 2006). Thus, a lambdoid phage through recombination may share some genes with some members and other genes with other members of the family. For example, 933W (Fig. 1) has the same $\mathrm{N}$ transcription protein and 
A
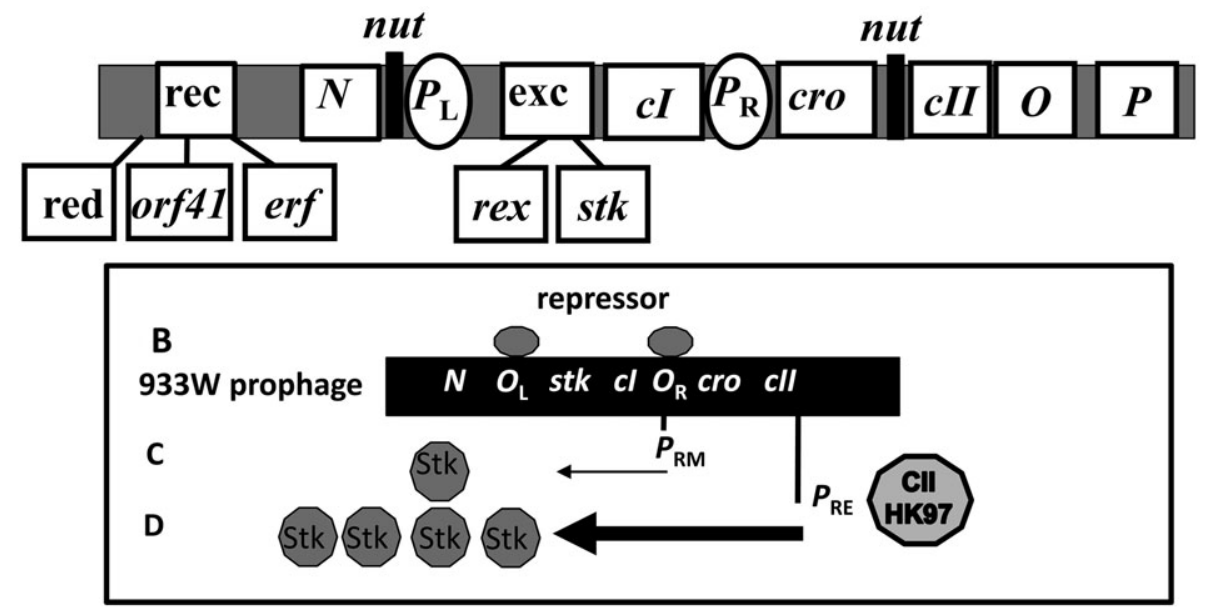

Cll proteins

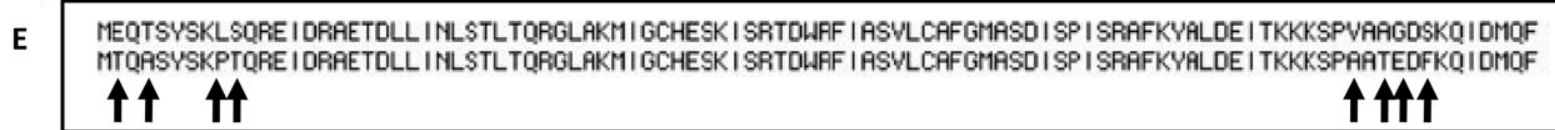

F $\quad P_{\mathrm{RE}}$ promoters $]_{\text {HK97 TTGCTGTAACTTGI }}^{933 \mathrm{~W} \text { TTGCTGTAACTTGC }}$

Fig. 1. Early regulatory region of lambdoid phages and control of Stk expression by infecting HK97.

A. Genetic map of early regulatory region of lambdoid phages showing position of selected genes. Shown are the similar locations (exc) of the rex gene of $\lambda$ and stk gene of 933W and the similar locations (rec) of the red genes of $\lambda$, erf gene of P22, and orf41s of HK97 and HK022 (not drawn to scale).

B. 933W $\mathrm{cl}$ operon showing position of stk and other genes relevant to this study (not drawn to scale).

C. Transcription of $\mathrm{cl}$ operon (includes stk) during lysogeny.

D. Action of HK97 Cll protein in stimulating transcription of $933 \mathrm{~W} \mathrm{Cl}$ operon.

E. Comparison of $933 \mathrm{~W}$ and HK97 Cll proteins (arrows indicate positions of codon differences).

F. Similarity of $P_{\mathrm{RE}}$ promoters of $933 \mathrm{~W}$ and HK97 (single nucleotide difference is underlined).

recognition sites (nut) flanking the $\mathrm{cl}$ repressor gene as another lambdoid phage, HK97 (Friedman and Court, 2001). However, these two phages have different $c /$ genes (Plunkett et al., 1999; Juhala et al., 2000). In contrast, HK97 and $\lambda$ have different $N$ genes and nut sites, but share the same $c /$ gene. However, HK97 and $\lambda$ both lack the stx2 genes and the gene of our interest stk, both found in 933W.

The stk gene encodes a eukaryotic-like tyrosine kinase (Plunkett et al., 1999; Tyler and Friedman, 2004). Although for many years thought to be limited to higher organisms, eukaryotic-like tyrosine as well as serine-threonine kinases have been identified in a number of bacteria (Kennelly and Potts, 1996; Leonard et al., 1998; Grangeasse et al., 2007; Pereira et al., 2011) as well as phage (Robertson et al., 1994). Because it is in the same operon as the $c /$ repressor gene, stk is expressed by the prophage; i.e., Stk is constitutively expressed at low levels from the $P_{\mathrm{RM}}$ promoter (Fig. $1 \mathrm{~B}$ and C) (Tyler and Friedman, 2004).

We report that a lysogen of $933 \mathrm{~W}$ through the activity of the Stk tyrosine kinase excludes infection by HK97 (Dhillon et al., 1980; Juhala et al., 2000). The 933W stk gene is positioned, like the rex exclusion genes of $\lambda$ (Howard, 1967), downstream of the $\mathrm{cl}$ gene (Fig. 1A). Although
Stk-directed protein phosphorylation is not observed in extracts of the 933W lysogen, significant phosphorylation is observed following infection by the excluded HK97 phage. An HK97 open reading frame, orf41, is required to activate the Stk expressed from the $933 \mathrm{~W}$ prophage that leads to exclusion of the infecting HK97 phage.

\section{Results}

\section{Exclusion of HK97 by the 933W prophage}

Efficiency of plating (eop) was used to obtain a quantitative measure of $\mathrm{HK} 97$ exclusion by the $933 \mathrm{~W}$ prophage. The E. coli K12 strain K37 served as the standard (Tables 1 and 2). A reduction in eop of approximately five logs was observed when titres of an HK97 lysate measured on lawns of K9675 (a derivative of K37 with a 933W prophage) and K37 were compared (Table 1). This exclusion is due to the tyrosine kinase activity of the 933W stk gene product (gp) as shown by eop measurements with strain K11515, a derivative of K9675 in which the 933W stk gene has a single codon change that eliminates Stk kinase activity (Tyler and Friedman, 2004). A comparison 
Table 1. Eop phage (titre on tested strain/titre on K37).

\begin{tabular}{|c|c|c|c|c|c|c|c|c|}
\hline Tested Strain & 933W prophage & stk allele & HK97 & HK022 & HK97 orf41-1 & HK97 $\Delta$ clk>cat & HK97 441 & HK97 $\Delta 41 \Delta c / k>c a t$ \\
\hline $\mathrm{K} 37^{\mathrm{b}}$ & - & - & 1 & 1 & 1 & 1 & 1 & 1 \\
\hline K9675 & + & wt & $\sim 10^{-5}$ & $0.5^{\mathrm{a}}$ & $\sim 1$ & $\sim 10^{-5}$ & $\sim 1$ & $\sim 1$ \\
\hline K11515 & + & K42R & $\sim 1$ & ND & ND & ND & ND & ND \\
\hline K10567 & + & stk-c-Myc & $\sim 10^{-4}$ & ND & ND & ND & ND & ND \\
\hline
\end{tabular}

a. Much smaller plaques on K9675.

b. Control.

See Experimental procedures for details.

ND, not done.

of HK97 titres on lawns of K37 and K11515 revealed no differences; i.e., the eop on K11515 using K37 as the standard was $\sim 1$. In addition, exclusion appears to be specific for HK97. A number of other phages tested were not excluded (Table 2). These included $\lambda$ (Hendrix et al., 1983), $\phi 80$ (Matsushiro, 1961), H-19B (Scotland et al., 1983; Huang et al., 1986) and $\lambda$-P22dis (a $\lambda$-P22 hybrid, see below) (Yamamoto et al., 1977) as well as T4rll (Benzer, 1955). All of those phages plate with an eop of $\sim 1$ on K11541, a derivative of $\mathrm{K} 9675$ in which a second exclusion system that affects some phages, including $\lambda$ and $\mathrm{H}-19 \mathrm{~B}$ (unpublished results, this laboratory) was deleted. An exception was HK022 (Dhillon and Dhillon, 1976), which was partially excluded (see below).

\section{HK97 infection and Stk-directed protein phosphorylation}

Tyrosine phosphorylation of proteins was examined by immunoblot analysis using an anti-phosphotyrosine antibody. To begin, we consider two limitations regarding the use of immunoblots in this paper. (1) A large number of proteins are phosphorylated by Stk and therefore the immunoblots are used to provide a measure of total protein phosphorylation without regard to individual proteins. (2) Although small differences in intensity in different regions of the immunoblots were observed from experiment to experiment, the overall patterns of large or small amounts of total phosphorylation were consistently observed. These small differences likely reflect the lack of specificity of Stk phosphorylation activity.

Our immunoblot analyses showed very little tyrosine phosphorylation in the uninfected 933W lysogen K9675 (Figs 2 and 4). However, HK97 infection of 933W lysogens K9675 and K10567 results in extensive tyrosine

Table 2. Eop (titre on K11541/titre on K37).

\begin{tabular}{ccccccc} 
HK97 & $\lambda$ & HK022 & $\lambda$-P22dis & H-19B & $\phi 80$ & T4rll \\
\hline$<10^{-4}$ & $\sim 1$ & $0.5^{\mathrm{a}}$ & $\sim 1$ & $\sim 1$ & $\sim 1$ & $\sim 1$ \\
\hline
\end{tabular}

a. Much smaller plaques on $\mathrm{K} 11541$.

See Experimental procedures for details. phosphorylation (Figs 2-5). This phosphorylation was shown to result from the kinase activity of Stk using K11515, the derivative of K9675 with a single base change at stk codon 42 that eliminates kinase activity (Tyler and Friedman, 2004). HK97 infection of this strain failed to direct protein phosphorylation (Fig. 2).

\section{Timing of Stk synthesis and protein phosphorylation following HK97 infection}

We followed Stk production and protein phosphorylation after HK97 infection of K10567, a lysogen in which the 933W prophage has a c-Myc tagged stk gene that retains close to wild-type function (Fig. 3 and Table 1). The tagged Stk was used because our anti-Stk antibody was ineffective at identifying Stk at levels expressed during infection with HK97. Stk levels were detected at $10 \mathrm{~min}$ post infection and preceded protein phosphorylation which was not observed until 15 min following infection. Significant phosphorylation was not observed until 20 min.
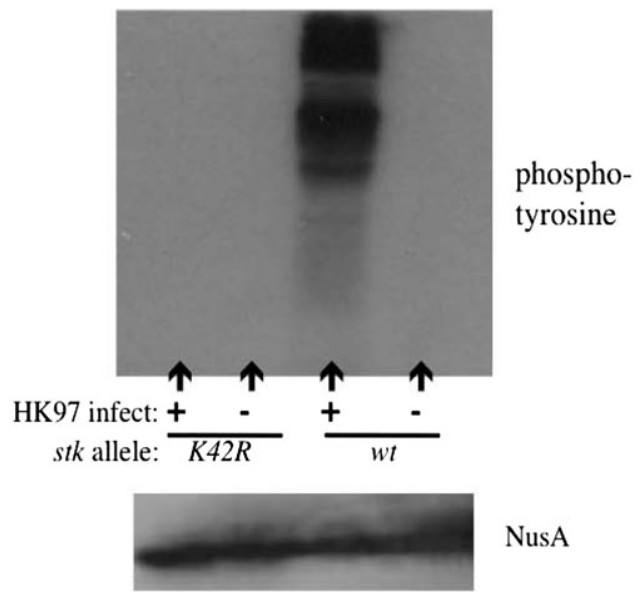

Fig. 2. Immunoblot showing Stk-directed tyrosine phosphorylation following infection of 933W lysogens with HK97. Strain K9675 $\left(s t k^{+}\right)$: lanes: 3 (infected) and 4 (uninfected). Strain K11515 (stkK42R): lanes: 1 (infected) and 2 (uninfected). NusA probed as a loading control. See Experimental procedures for details. 


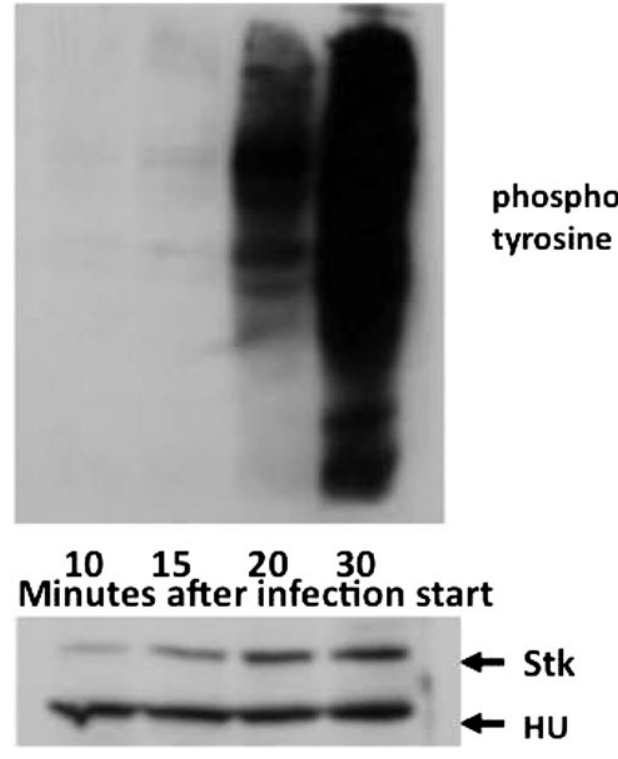

Fig. 3. Timing of Stk production and protein phosphorylation following HK97 infection of 933W lysogen (K10567). Ten-millilitre aliquots were removed at indicated times and samples prepared for immunoblot analysis. The commercial anti-c-Myc used to identify Stk was fortuitously contaminated with anti-HU, which served as a loading control. See Experimental procedures for details.

\section{HK97 Cll protein and Stk synthesis}

Because stk is in the same 933W operon as $c /$ repressor (Tyler et al., 2004), we reasoned that the HK97 function responsible for activating Stk both in its roles of excluding HK97 and promoting protein phosphorylation would likely increase transcription of $\mathrm{cl}$ and genes in that operon from the low levels expressed from the 933W prophage,

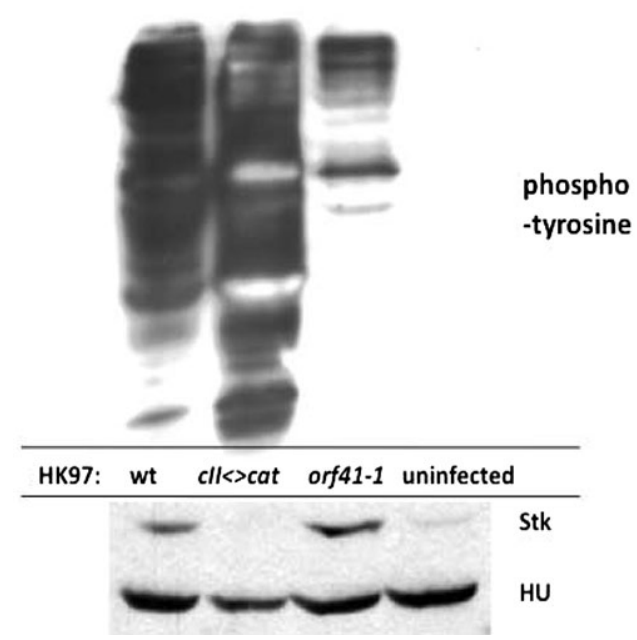

Fig. 4. Immunoblot showing Stk-directed protein phosphorylation and production following infection of K10567 with HK97 variants and an uninfected control (K10567). The commercial anti-c-Myc used in this experiment as discussed in the legend to Fig. 3 allowed identification of Stk and HU. See Experimental procedures for details. autogenously regulated at $P_{\mathrm{RM}}$ by repressor (Ptashne et al., 1976) (Fig. $1 \mathrm{~B}-\mathrm{D})$. The $\lambda$ Cll protein and its homologues in other lambdoid phage act at promoters, $P_{\mathrm{RE}}$ (Fig. 1D) to direct high-level transcription of $C l$ (Reichardt and Kaiser, 1971; Wulff and Rosenberg, 1983; Ho and Rosenberg, 1988). This led us to hypothesize that the HK97 and 933W Cll proteins might be so similar that the Cll protein expressed by the infecting HK97 binds at the $P_{\mathrm{RE}}$ of the 933W prophage directing high levels of transcription of the $933 \mathrm{~W} \mathrm{cl}$ operon with its downstream stk gene (Fig. 1D). Comparison of $\mathrm{Cll}$ and $P_{\mathrm{RE}}$ sequences of HK97 and 933W showed they are indeed very similar (Fig. 1E and F).

Infection with HK97 resulted in a substantial increase in Stk, while a mutant HK97 with a deletion substitution for the cll gene, HK97clk $>$ cat, failed to show evidence of increased synthesis (Fig. 4). This is consistent with our hypothesis that $\mathrm{HK} 97 \mathrm{Cll}$ acts to direct high-level expression of Stk. Surprisingly, despite the failure to increase synthesis of Stk, HK97 clk>cat was excluded by the 933W prophage (Table 1) and directed levels of protein phosphorylation comparable to those directed by the wildtype HK97 (Fig. 4). Hence, HK97-directed protein phosphorylation and associated exclusion do not require highlevel expression of Stk.

\section{Identifying the HK97 function(s) required for exclusion}

To identify the HK97 function(s) involved in exclusion by Stk, we selected for and isolated a mutant of HK97 not excluded by the $933 \mathrm{~W}$ prophage (Table 1). Comparison of
A

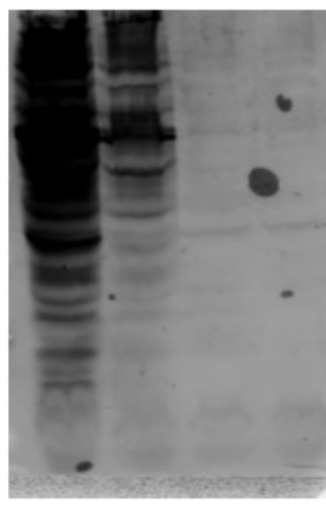

HK97 HK022 $\lambda$-P22 C

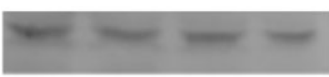

B

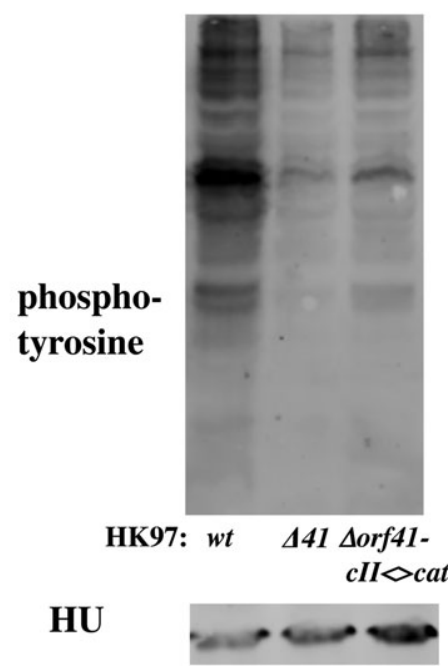

Fig. 5. Immunoblots showing protein phosphorylation by Stk following infection of K10567 by phage with orf41-like genes. HU served as loading control.

$A$ and $B$. Comparison following infections with different phages and uninfected control (C). 
the DNA sequences of the mutant and parental HK97 phages showed a difference in only one gene, orf 41 . This gene has partial sequence homology with the erf gene of phage P22 (Juhala et al., 2000; Hendrix, 2006). The P22 erf gene encodes a recombination function essential for phage growth in a rec- host (Botstein and Matz, 1970). The genome position of orf41 is analogous to that of erf (Botstein and Matz, 1970) as well as the $\lambda$ red recombination genes (Oppenheim and Court, 1983) (Fig. 1A). Taken together, these observations suggest that orf41 encodes a recombination function. The HK97 orf41 mutation allowing growth in a 933W lysogen causes a frameshift resulting in premature translation termination. Based on this observation, we have named the mutant phage HK97orf41-1. DNA sequencing showed that the cll gene in this mutant phage is wild-type. Infection with HK97orf41-1 results in increased levels of Stk comparable to those observed in the wild-type infection (Fig. 4). Notwithstanding the elevated levels of Stk, infection with HK97orf41-1 results in a significantly lower level of protein phosphorylation (Fig. 4).

To confirm that orf41 is necessary for the Stk-directed exclusion by the $933 \mathrm{~W}$ prophage, we constructed a derivative of HK97 with orf41 deleted. This phage, HK97 $\Delta$ orf41, exhibits the same phenotypes as HK97orf41-1 during infection of a 933W lysogen; i.e., is not excluded and promotes only low observable levels of protein phosphorylation by Stk (Table 1 and Fig. 5B).

With these results in mind, we return to the observation that infection of a $933 \mathrm{~W}$ lysogen with $\mathrm{HK} 97 \mathrm{clk}<\mathrm{cat}$ mutant results in phosphorylation levels similar to those seen with wild-type HK97 infection without a concomitant increase in Stk levels. This led us to the question whether the cll mutant relies on a different pathway for activating Stk, one independent of gpOrf41. To answer this question, we constructed HK97 $\Delta$ orf41-clk>cat. This phage exhibits precisely the same phenotypes as HK97 $\Delta$ orf41; on infection of a $933 \mathrm{~W}$ lysogen shows no defect in plating (Table 1) and exhibits the same weak protein phosphorylation (Fig. 5B). Hence, the gporf41 is responsible for the exclusion and the significant level of protein phosphorylation observed following infection of the 933W lysogen with HK97clk>cat.

\section{Other phages and protein phosphorylation}

To further determine if there is a correlation between the effect of Stk on exclusion and protein phosphorylation, we examined protein phosphorylation following infection with a weakly excluded phage, HK022, and one that is not excluded by this system, $\lambda$-P22 dis, a hybrid phage that includes the P22 erf gene (Yamamoto et al., 1978). Neither of these phages is affected by the second 933W exclusion system discussed above (unpublished results, this laboratory). Results of these experiments are consistent with the previously discussed eop results (Table 1). Infections with $\lambda$-P22dis failed to result in observable protein phosphorylation (Fig. 5A). Infection with HK022, whose orf41 shares extensive homology with HK97 orf41 (Juhala et al., 2000), results in protein phosphorylation. However, the levels are less than those observed following HK97 infection (Fig. 5A).

\section{Functional relation of the orf41and P22 erf gene products}

The HK97 orf41 was annotated as erf-like (Juhala et al., 2000) based on the observation that there is significant identity in its $5^{\prime}$ region with the $5^{\prime}$ region of the $\mathrm{P} 22$ erf gene (Hendrix, 2006). The P22 erf (essential recombination function) gene was so named because its product is required for phage growth in a RecA deficient host. Under that condition, Erf is required for circularization of the terminally redundant $\mathrm{P} 22$ genome, a step required for phage growth (Botstein and Matz, 1970). We found that HK97 $\Delta$ orf41 growth is not significantly reduced in a recA mutant, K3701 (data not shown) providing compelling evidence that gpOrf41, although possibly encoding a recombination function, is not essential for HK97 growth in the absence of host recombination activity. The infecting HK97 DNA most likely circularizes by a mechanism similar to that of $\lambda$ (Yarmolinsky, 1971).

\section{Autophosphorylation and Stk stability}

We examined Stk stability and autophosphorylation using strains K11569, encoding the c-Myc-tagged stk gene, and $\mathrm{K} 11580$, isogenic except for the stkK42R mutation that eliminates kinase activity. These strains have these stk genes cloned downstream of the $\lambda P_{\mathrm{L}}$ promoter in the TUC 01 strain (Datta et al., 2008) (Fig. 6A). This places the stk genes under control of the strong $P_{\mathrm{L}}$ promoter (Shatzman et al., 1983) and temperature sensitive $\lambda$ Cl857 repressor (Sussman and Jacob, 1962), which denatures at high temperature (e.g. $\left.42^{\circ}\right)$ and renatures at low temperature (Fig. 6A). Hence, Stk will only be expressed during the time the bacteria are grown at high temperature.

The following briefly outlines the experimental design we used to examine Stk stability (see Experimental procedures for details). Bacteria were grown at $30^{\circ}$ to early log phase, where Stk is not expressed, shifted for $20 \mathrm{~min}$ to $42^{\circ}$, where Stk is expressed, and, following treatment in an ice bath to insure that repressor renatures, shifted to $30^{\circ}$ where again Stk is not expressed. Stk levels and physical properties were measured by immunoblot from aliquots removed at times indicated in Fig. 6B after the shift back to $30^{\circ}$. 
A
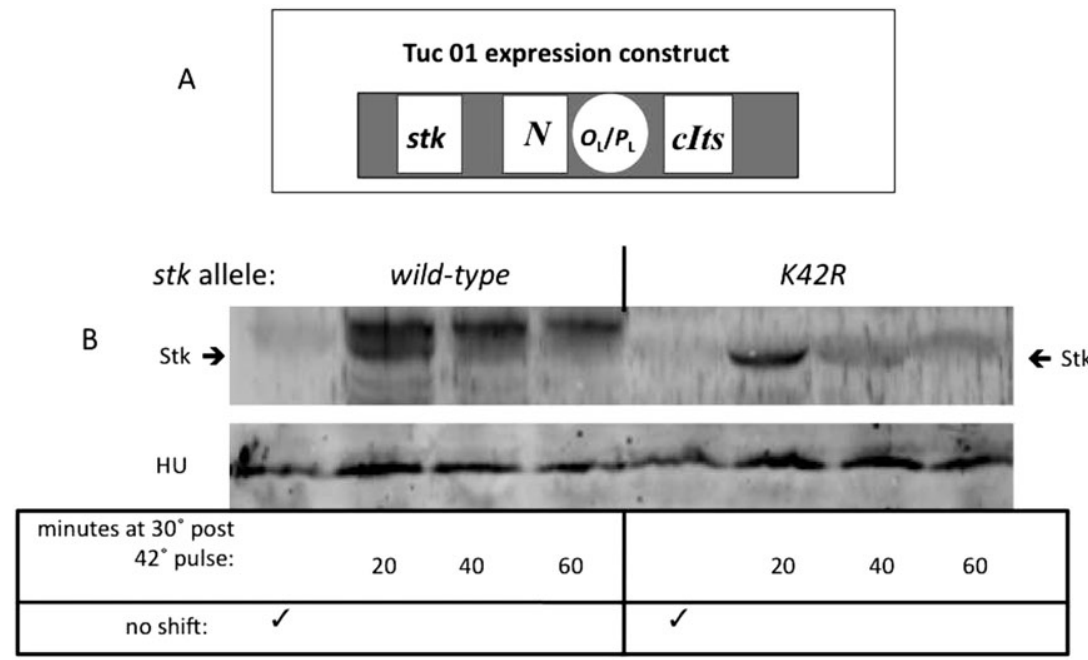

Fig. 6. In vivo stability of Stk.

A. Relevant features of chromosomally located TUC 01 construct (Datta et al., 2008); the stk gene is placed downstream of the $\lambda P_{\mathrm{L}}$ promoter under control of the temperature sensitive renaturable $\mathrm{Cl} 857$ repressor. B. Immunoblot showing fate of wild-type Stk, strain K11569 (left) and K42R mutant, strain K11580 (right). Stk was expressed for 20 min by a shift to $42^{\circ}$, where $\mathrm{Cl} 857$ repressor is denatured (Sussman and Jacob, 1962),

followed by shift to $30^{\circ}$ for indicated times, where renatured $\mathrm{Cl} 857$ repressor blocks new synthesis of Stk.
The results of the experiment are shown in Fig. 6B. Two differences are apparent between Stk in the strains expressing wild-type (K11569) and those expressing K42R (K11580) Stk. First, at 20 min post shift, wild-type Stk appears as two distinct, but closely migrating bands. In contrast, Stk-K42R appears as a single band that comigrates with the lower, faster migrating, wild-type Stk band. This suggests that the slower migrating band from the wild-type results from autophosphorylation. This is consistent with our previous study showing evidence of Stk autophosphorylation (Tyler and Friedman, 2004). Second, the faster-migrating (non-autophosphorylated) bands of both wild-type and Stk-K42R origin are relatively unstable. By 40 min post shift to $30^{\circ}$, loss of Stk is substantial in isolates from $\mathrm{K} 11580$. In contrast, at $60 \mathrm{~min}$ post shift there is still significant Stk of the slower migrating species from K11569. From the fact that the slower migrating band is the stable form, we conclude that autophosphorylation contributes to Stk stability.

\section{High expression of Stk and bacterial viability}

High levels of Stk can be lethal for E. coli as shown by studies with the TUC 01 stk construct (Fig. 6A). Cultures of K11569 (contains the TUC 01 stk construct) and K11580 (contains the TUC 01 stk-k42R construct) were grown at $30^{\circ}$. Each culture was divided into two aliquots; one aliquot maintained at low temperature while the other pulsed for $30 \mathrm{~min}$ at $42^{\circ}$. As discussed, the shift to $42^{\circ}$ denatures the $\lambda \mathrm{Cl} 857$ ts repressor allowing expression of Stk or Stk-K42R. Following the pulse, aliquots were cooled in ice to insure repressor renaturation. Dilutions of each aliquot were spread on LB plates that were incubated overnight at $32^{\circ}$ (a temperature at which the $\mathrm{Cl} 857$ repressor is stable) to measure viable bacterial count. Under these conditions the construct expressing func- tional Stk shows extensive protein phosphorylation (data not shown).

Results of the experiment are shown in Table 3. Comparing growth of the two strains at $30^{\circ}$, we find both had the same titre, $\sim 2 \times 10^{8}$. However, at $42^{\circ}$, where the bacteria grow faster, the titre of the bacteria with the stk-K42R allele is greater than 50 -fold higher than the titre of the bacteria with the wild-type stk allele (Table 3). Moreover, the survivors of $42^{\circ}$ growth took longer to recover. We conclude that expression of Stk can be lethal to the bacterium. Second, we infer that the lethal effect is due to protein phosphorylation. However, that conclusion must be tempered considering the results discussed above that less Stk is present in bacteria expressing Stk-K42R.

\section{Discussion}

Bacterial protein kinases, like those in higher organisms, modulate activity of many proteins including a large number of those involved in regulatory processes (Krell et al., 2010). Many of these are histidine kinases (Mascher et al., 2006; Pereira et al., 2011). Bacterial tyrosine kinases are rare (Grangeasse et al., 2007), with eukaryotic-like tyrosine kinases even less abundant (Leonard et al., 1998). Hence, it was surprising to find a

Table 3. Survival following 20 min expression of Stk.

\begin{tabular}{llll} 
Strain & stk allele & Shift to $42^{\circ a}$ & Titre \\
\hline K11569 & wt & No & $2 \times 10^{8}$ \\
K11569 & wt & Yes & $5 \times 10^{6 b}$ \\
K11580 & K42R & No & $2 \times 10^{8}$ \\
K11580 & K42R & Yes & $3 \times 10^{8}$ \\
\hline
\end{tabular}

a. Stk only expressed during $20 \mathrm{~min}$ at $42^{\circ}$.

b. Survivors took longer to recover.

See Experimental procedures for details. 
eukaryotic-like tyrosine kinase encoded in the genome of a phage. Although phage-encoded protein kinases are uncommon, one of the best studied is the serine/threonine kinase of phage T7 and, like Stk, the T7 kinase phosphorylates a large number of proteins (Robertson et al., 1994).

Because Stk is similar to eukaryotic tyrosine kinases, is found in the same phage genome as the stx genes, and the prophage is found in pathogenic EHEC strains (e.g. O157:H7), it is plausible to consider that Stk is a virulence factor. Moreover, eukaryotic-like serine/threonine kinases play important roles in virulence of a number of Grampositive pathogens (Burnside and Rajagopal, 2011). However, we have not found any supporting evidence for this idea, nor had we been able to identify a phenotype for Stk until we found that Stk was responsible for the exclusion of HK97 by the $933 \mathrm{~W}$ prophage.

Based on the studies reported here, we can conclude that the tyrosine kinase activity of Stk is responsible for exclusion of phage HK97 by the $933 \mathrm{~W}$ prophage. Less obvious, however, is the mechanism that activates Stk to catalyse protein phosphorylation. Curiously, even though HK97 infection results in increased production of Stk, this increased production is neither necessary nor sufficient for HK97-directed stimulation of Stk phosphorylation activity. Key to understanding the role of HK97 in this process are experiments with HK97 mutants. Infection with an HK97 lacking $c / l$ (cll<>cat) showed that increased levels of Stk are not necessary and HK97 derivatives missing orf41 showed that the increased levels, similar to those observed with HK97 wild-type infection, are not sufficient. The observation that $\lambda$-P22dis, which is not excluded, does not activate Stk confirms that HK97 plays an essential role in its own exclusion during infection of the $933 \mathrm{~W}$ lysogen.

The $933 \mathrm{~W}$ prophage through action of its stk gene product is not sui generis in exhibiting specificity in excluding another lambdoid phage. The HK022 prophage through action of its nun gene product only excludes the few lambdoid phages that have the $N$ gene of $\lambda$ (Robert et al., 1987).

Although infection of the 933W lysogen with either HK97orf41-1 or HK974orf41 fails to result in exclusion, the infections do result in some tyrosine phosphorylation, albeit significantly reduced from that observed with HK97 infection. This information does not lead us to question whether orf41 is necessary for exclusion, but it does raise the possibility that the orf41 gene product may not be sufficient for exclusion; an additional function expressed by HK97 may be required for effective exclusion.

How might proteins act to alter Stk to a form that catalyses extensive protein phosphorylation and act to exclude HK97? Our studies indicate that autophosphorylation plays an essential role. Although an activator protein (e.g. gp41) is not required for autophosphorylation when Stk is at high concentration, it is required for activity and presumably autophosphorylation when Stk is at the low concentration levels of the lysogen. Such requirements for activation are not unique to Stk. By structure and function Stk is grouped with the extensively studied eukaryotic kinases (Plunkett et al., 1999; Tyler and Friedman, 2004). Consistent with our observations regarding Stk, activity of many of these kinases require autophosphorylation (Smith et al., 1993) that occurs either through dimerization or interaction with effector proteins (Roskoski, 2004; Pike et al., 2008). We propose that the HK97 gp41 serves as an activator stimulating Stk autophosphorylation resulting in exclusion. We have not yet identified any specific proteins whose phosphorylation causes exclusion, nor have we ruled out the possibility that the large number of proteins phosphorylated by Stk leads to a loss of cellular activity necessary for phage production. However, our finding that a short exposure to high levels of Stk affects bacterial viability is consistent with such a possibility.

Stimulation of Stk autophosphorylation is not limited to gp41 and possibly another protein encoded by HK97. In a previous in vitro study, we showed that histone protein II, which is phosphorylated by Stk, stimulates Stk autophosphorylation (Tyler and Friedman, 2004). However, in the same study we found that myelin basic protein, which also is phosphorylated by Stk, does not stimulate autophosphorylation. We postulate that the high levels of Stk synthesized in the isolation of rStk (an epitope tagged, but active Stk) allow dimerization resulting in incomplete but sufficient auto-phosphorylation of Stk to stimulate enzymatic activity. However, interaction with histone protein II, presumably similar to the interaction with gp41, supports further autophosphorylation. This raises the question as to whether there is a condition(s) in which a bacterial protein is expressed that stimulates Stk activity. Two observations make such a scenario plausible. First, Stk is constitutively expressed by the $933 \mathrm{~W}$ prophage (Tyler and Friedman, 2004) and second, these constitutive levels are sufficient to promote significant protein phosphorylation in the presence of the gp41. However, the experiments with the TUC 01 construct show that when Stk is expressed at high levels it can autophosphorylate.

Our observation that expression of activated Stk interferes with cell growth suggests a possible function similar to toxin-antitoxin (programmed cell death) systems (Yarmolinsky, 1995; Van Melderen \& Saavedra De Bast, 2009). As discussed above, such a system has been implicated in protection against phage infection (Fineran et al., 2009). In these systems, the toxin, which is inhibitory to cell growth, is held in check by the antitoxin. In the usual case, the toxin is more stable than the antitoxin and thus when concentrations of antitoxin are reduced below those of the toxin, cell death or stasis ensues. Many roles 
Table 4. Relevant genotype and source of bacteria.

\begin{tabular}{|c|c|c|}
\hline Strain & Relevant genotype & Reference/source \\
\hline K37 & K12 lab strain & This lab \\
\hline TUC 01 & 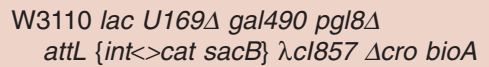 & Don Court (Datta et al., 2008) \\
\hline K9675 & K37 (933W) & Tyler and Friedman (2004) \\
\hline K11515 & K37 (933W stk-K42R) & This lab \\
\hline K10567 & K37 (933W stk-c-Myc) & This lab \\
\hline K11541 & K37 (933W $\Delta$ ori $\Delta$ LO095) & This lab \\
\hline K11569 & TUC 01 (int-cIII)<>stk-c-Myc & This lab \\
\hline K11580 & TUC 01 (int-cllI)<>stkK42R-c-Myc & This lab \\
\hline K2701 & $\mathrm{K} 37(\Delta s r l-r e c A)$ & This lab \\
\hline
\end{tabular}

have been proposed for these systems, e.g. plasmid maintenance by destroying bacteria that lose the plasmid, population survival by death of the many to sustain the few, and growth stasis, called persistence, of a fraction of the population resulting in escape from action of harmful agents such as antibiotics. A case in point is HipA, the toxin in the HipA-B system, which like Stk is a eukaryoticlike kinase, but in this case a serine kinase (Correia et al., 2006; Schumacher et al., 2009). Unlike Stk, HipA phosphorylation appears directed to one protein, transcription factor EF-Tu, in this way inhibiting protein synthesis and establishing a surviving (persistent) subpopulation in the presence of antibiotic. Stk differs from classical toxins by remaining quiescent until activated, suggesting a pretoxin whose activity requires not loss of an antitoxin but gain of a protoxin. If Stk serves in some type of toxin role, we speculate that the bacterium encodes the Stk activator, protoxin, which is expressed under specific stress conditions at levels that limit bacterial growth of a subpopulation until more favourable conditions are encountered. The protoxin that activates Stk causing aborted HK97 infection is the gp41 made by the infecting HK97. This leads to a dead end for HK97 blocking further growth and in this way protecting the remaining bacterial population from further infection.

Here we list a few unanswered questions we consider most compelling. Is the origin of Stk eukaryotic? Is phage exclusion driving selection or is that activity a fortuitous addition to Stk's main activity? If the latter, what is that activity? The immunoblots showed a large number of proteins phosphorylated, raising the following question: does this promiscuity have physiological significance? Is phosphorylation of a specific protein(s) the cause of exclusion or is it the wide range of proteins phosphorylated?

\section{Experimental procedures}

\section{Bacteria and phage}

See Tables 4 and 5.

\section{Media}

LB broth, LB sucrose, top agar and TB have previously been described (Livny and Friedman, 2004). Antibiotics were used at the following concentrations: ampicillin $100 \mu \mathrm{g} \mathrm{ml}^{-1}$, chloramphenicol $8 \mu \mathrm{g} \mathrm{ml}^{-1}$ and kanamycin $30 \mu \mathrm{g} \mathrm{ml}^{-1}$.

\section{Phage infection}

Cultures were grown overnight in LB maltose $(0.4 \%)$ and diluted into LB maltose, grown to $\sim 10^{8} \mathrm{ml}^{-1}$, and centrifuged. Sedimented bacteria were resuspended in buffer (yielding a 10-fold concentration) appropriate for adsorption of the phage: for HK97, 10 mM Tris pH 8.0 and for all other phages,

Table 5. Relevant information and source of phage.

\begin{tabular}{lll} 
Phage & Relevant information & Source/reference \\
\hline HK97 & Wild-type & Roger Hendrix (Dhillon et al., 1980) \\
HK97cll<>cat & Deletion substitution of cll & This lab \\
HK97orf41-1 & Plates on 933W lysogen & This lab \\
$\lambda$ & $\lambda+$ & NIH collection \\
HK022 & Lambdoid phage & Robert Weisberg (Dhillon and Dhillon, 1976) \\
$\lambda-P 22 d i s$ & $\lambda$ P22 hybrid & Yamamoto et al. (1977) \\
H-19B & Lambdoid phage & O'Brien et al. (1984) \\
T4rll & Does not plate on $\lambda$ lysogen due to action of Rex & Robert Greenberg (Benzer, 1955) \\
HK97 orf41 & In frame deletion & This lab \\
HK97 $\Delta$ orf41-cll<>cat & Double mutant & This lab \\
$\phi 80$ & Lambdoid phage & NIH collection
\end{tabular}


$0.01 \mathrm{M} \mathrm{Mg}^{++}$. Phage were added at a multiplicity (moi) of $\sim 5$ and allowed to adsorb for $10 \mathrm{~min}$. Infected bacteria were microfuged. Supernatants were titred for unadsorbed phage (allowing calculation of per cent adsorbed) and sediments were resuspended in $10 \mathrm{ml} \mathrm{LB}$ and grown for $20 \mathrm{~min}$ at $37^{\circ}$. Infected bacteria and uninfected controls were centrifuged and sedimented cells resuspended in $1 \mathrm{ml}$ of buffer, microfuged, and prepared for electrophoresis as described below.

\section{Timing of Stk synthesis and protein phosphorylation after HK97 infection}

Following adsorption, infected bacteria were resuspended in LB and grown at $37^{\circ}$. Ten-millilitre aliquots were removed at times indicated in Fig. 3 for immunoblot analysis as described below.

\section{Immunoblots}

Procedure was essentially as previously described (Tyler and Friedman, 2004). Cell extracts were prepared in two ways. (1) Samples were resuspended in $90 \mu \mathrm{l}$ of loading buffer (Sambrook et al., 1989), boiled for $20 \mathrm{~min}$, supernatants were collected following a $10 \mathrm{~min}$ spin in the microfuge, and either used immediately or frozen for later use. (2) Bacteria were sonicated, filtered and concentrated prior to preparation for gel loading as above (Fig. 2). Proteins were separated using SDS-PAGE electrophoresis. Lanes were overloaded with protein to maximize sensitivity of assay.

Stk levels were assessed using either of two antisera: (1) a rabbit antiserum raised against Stk protein (Tyler and Friedman, 2004), which is only effective when Stk is overexpressed, and (2) a commercially (Santa Cruz Biotechnologies) obtained mouse HRP conjugated anti-c-Myc that identifies Stk that was c-Myc tagged and is effective when Stk-c-Myc is expressed at lower levels. The tagged Stk maintained the observed phenotypes of excluding HK97 (only slightly less effective, Table 1) and showed similar protein phosphorylation following HK97 infection. Tyrosine phosphorylation was assessed using an HRP conjugated anti-phosphotyrosine antibody (Santa Cruz Biotechnology). HU loading control was detected using HRP conjugated anti-HU antibody as a known contaminant of an early preparation of the commercial anti-c-Myc antibody (Santa Cruz Biotechnologies) or anti-HU kindly supplied by Dr Sankar Adhya. Anti-NusA was from this laboratory. Where required, blots were treated with secondary alkaline phosphatase conjugated donkey anti-rabbit antiserum (GE Healthcare UK Limited). Bands were identified using ECL (General Electric) and viewed using either film or the Typhoon Trio Variable Mode Imager.

\section{Genetic constructs}

The $\lambda$ Red system (recombineering) was used to make all genetic substitutions and changes (Datsenko and Wanner, 2000; Court et al., 2002). When a selection was not available, a two-step procedure was used; first a double-stranded catsacB cassette (CSB) was inserted at the designed location
(Yu et al., 2000) and subsequently replaced with doublestranded DNA or single-stranded oligonucleotide with the designed change.

The TUC 01 strain (a derivative of SIMD30) (Datta et al., 2008), used for controlled expression of Stk, has a portion of a $\lambda$ prophage that includes the $c / 857$ (ts) gene and the genes of the $\lambda P_{\mathrm{L}}$ operon under control of the strong $\lambda P_{\mathrm{L}}$ promoter. This promoter has $8-10$ times the strength of the $P_{\text {lac }}$ (Shatzman et al., 1983). Inserted in the int gene is a CSB. The defective prophage itself can supply the Red functions for recombineering when shifted to $42^{\circ}$ as in a standard recombineering reaction (Datta et al., 2008). The Stk construct, which has the entire region from int through clll replaced with the stk gene (Fig. 6A), was obtained by selection on LB sucrose plates at $42^{\circ}$.

PCR and DNA sequencing were used to confirm that constructs conformed to the original design. Sequencing to confirm constructs and mutants was performed by the University of Michigan DNA Sequencing Core.

Sequence of the HK97 mutant determined to have the orf41-1 mutant was performed at the University of Pittsburgh (Pittsburgh Phage Institute). A comparison of the DNA sequence of the mutant HK97 with the published HK97 sequence alerted us to a number of orfs possibly indicating mutant genes. Each of the identified orfs from both the mutant and the parental HK97 from our laboratory were sequenced by the University of Michigan Sequencing Core facility. Comparison of these sequences revealed that only the orf41 pair differed.

\section{Assessing stability of Stk (Fig. 6)}

Cultures of K11569 and K11580 were grown to early log phase at $30^{\circ}$ in LB. Ten-millilitre aliquots of each were maintained at $30^{\circ}$ with shaking for the duration of the experiment and then prepared for immunoblot analysis. The rest $(45 \mathrm{ml})$ of the two cultures were shifted to and maintained at $42^{\circ}$ for 20 min with shaking, cooled in an ice bath to renature ts repressor, and placed at $30^{\circ}$ with shaking. Ten-millilitre aliquots of the heat-treated aliquots were removed at $20 \mathrm{~min}$ intervals following the shift back to $30^{\circ}$ and prepared for immunoblot analysis. This procedure provided a means to assess the in vivo stability of Stk; i.e., the Stk in cultures subsequently grown at $30^{\circ}$ largely will have been synthesized during the prior incubation at $42^{\circ}$.

\section{Measuring of exclusion (eop)}

Dilutions of phage were added to $2.5 \mathrm{ml}$ of top agar with $100 \mu \mathrm{l}$ of overnight culture of K37 and plated on TB plates that were incubated at $37^{\circ}$. The efficiency of plating (eop) is determined by dividing the phage titre on lawns of the tested strain by the titre on lawns of the control strain (K37).

\section{Survival following expression of Stk}

$\mathrm{K} 11569$ and $\mathrm{K} 11580$ were grown to early log phase at $30^{\circ}$ and each divided into two aliquots. One aliquot of each culture was kept a $30^{\circ}$ and the other shifted to $42^{\circ}$ for $30 \mathrm{~min}$. 
Following the shift, cultures were cooled on ice and dilutions were plated on LB plates that were incubated at $32^{\circ}$.

\section{Acknowledgements}

The authors thank the following: Steve King, Wesley Dunnick, Katherine Spindler, Phillip Lapinski, Syed Monen Rizvi, Nicole Koropatkin, Kathy Spindler, Natasha Del Cid, Chris Alteri and John Collins for technical consultations; Michael Imperiale and Don Court for helpful suggestions; Don Court, N. Cary Engleberg, Graham Hatfull and Victor DiRita for critical reading of the manuscript; and Sankar Adhya for anti-HU antibody. Work was supported in part by Public Health Service grant Al11459-10.

\section{References}

Ackermann, M., Stecher, B., Freed, N.E., Songhet, P., Hardt, W.D., and Doebeli, M. (2008) Self-destructive cooperation mediated by phenotypic noise. Nature 454: 987-990.

Arber, W. (1971) Host-controlled variation. In The Bacteriophage Lambda. Hershey, A.D. (ed.). Cold Spring Harbor, NY: Cold Spring Harbor Laboratory, pp. 83-96.

Benzer, S. (1955) Fine Structure of a Genetic Region in Bacteriophage. Proc Natl Acad Sci USA 41: 344-354.

Benzer, S. (1966) Adventures in the rll region. In Phage and the Origins of Molecular Biology. Cairns, J., Stent, G.S., and Watson, J.W. (eds). Cold Spring Harbor, NY: Cold Spring Harbor Laboratory, pp. 157-165.

Botstein, D., and Matz, M.J. (1970) A recombination function essential to the growth of bacteriophage P22. $\mathrm{J} \mathrm{Mol} \mathrm{Biol}$ 54: 417-440.

Brouns, S.J., Jore, M.M., Lundgren, M., Westra, E.R., Slijkhuis, R.J., Snijders, A.P., et al. (2008) Small CRISPR RNAs guide antiviral defense in prokaryotes. Science 321: 960-964.

Burnside, K., and Rajagopal, L. (2011) Aspects of eukaryoticlike signaling in Gram-positive cocci: a focus on virulence. Future Microbiol 6: 747-761.

Campbell, A. (1994) Comparative molecular biology of lambdoid phages. Annu Rev Microbiol 48: 193-222.

Campbell, A., and Botstein, D. (1983) Evolution of Lambdoid Phages. In Lambda II. Hendrix, R.W., Roberts, J.W., Stahl, F.W., and Weisberg, R.A. (eds). Cold Spring Harbor, NY: Cold Spring Harbor Laboratory Press, pp. 365-380.

Chopin, M.C., Chopin, A., and Bidnenko, E. (2005) Phage abortive infection in lactococci: variations on a theme. Curr Opin Microbiol 8: 473-479.

Correia, F.F., D'Onofrio, A., Rejtar, T., Li, L., Karger, B.L., Makarova, K., et al. (2006) Kinase activity of overexpressed HipA is required for growth arrest and multidrug tolerance in Escherichia coli. J Bacteriol 188: 83608367.

Court, D.L., Sawitzke, J.A., and Thomason, L.C. (2002) Genetic engineering using homologous recombination. Annu Rev Genet 36: 361-388.

Datsenko, K.A., and Wanner, B.L. (2000) One-step inactivation of chromosomal genes in Escherichia coli K-12 using PCR products. Proc Natl Acad Sci USA 97: 6640-6645.

Datta, S., Costantino, N., Zhou, X., and Court, D.L. (2008) Identification and analysis of recombineering functions from Gram-negative and Gram-positive bacteria and their phages. Proc Natl Acad Sci USA 105: 1626-1631.

Dhillon, T.S., and Dhillon, E.K. (1976) Temperate coliphage HK022. Clear plaque mutants and preliminary vegetative map. Jpn J Microbiol 20: 385-396.

Dhillon, E.K., Dhillon, T.S., Lai, A.N., and Linn, S. (1980) Host range, immunity and antigenic properties of lambdoid coliphage HK97. J Gen Virol 50: 217-220.

Duckworth, D.H., Glenn, J., and McCorquodale, D.J. (1981) Inhibition of bacteriophage replication by extrachromosomal genetic elements. Microbiol Rev 45: 52-71.

Fineran, P.C., Blower, T.R., Foulds, I.J., Humphreys, D.P., Lilley, K.S., and Salmond, G.P. (2009) The phage abortive infection system, ToxIN, functions as a protein-RNA toxinantitoxin pair. Proc Natl Acad Sci USA 106: 894-899.

Friedman, D.I., and Court, D.L. (2001) Bacteriophage lambda: alive and well and still doing its thing. Curr Opin Microbiol 4: 201-207.

Gottesman, S. (1998) Protecting the neighborhood: extreme measures. Proc Natl Acad Sci USA 95: 2731-2732.

Grangeasse, C., Cozzone, A.J., Deutscher, J., and Mijakovic, I. (2007) Tyrosine phosphorylation: an emerging regulatory device of bacterial physiology. Trends Biochem Sci 32: 86-94.

Hendrix, R.W. (2006) Bacteriophage $\lambda$ and its genetic neighborhood. In The Bacteriophages. Calendar, R. (ed.). Oxford: Oxford University Press, pp. 409-447.

Hendrix, R.W., Roberts, J.W., Stahl, F.W., and Weisberg, R.A. (1983) Lambda II. Cold Spring Harbor, NY: Cold Spring Harbor Laboratory.

Ho, Y.S., and Rosenberg, M. (1988) Structure and function of the transcription activator protein cll and its regulatory signal. In The Bacteriophages. Calendar, R. (ed.). New York: Plenum Press, pp. 725-756.

Howard, B.D. (1967) Phage lambda mutants deficient in r-II exclusion. Science 158: 1588-1589.

Huang, A., de Grandis, S., Friesen, J., Karmali, M., Petric, M., Congi, R., and Brunton, J.L. (1986) Cloning and expression of the genes specifying Shiga-like toxin production in Escherichia coli H19. J Bacteriol 166: 375-379.

Juhala, R.J., Ford, M.E., Duda, R.L., Youlton, A., Hatfull, G.F., and Hendrix, R.W. (2000) Genomic sequences of bacteriophages HK97 and HK022: pervasive mosaicism in the Lambdoid phages. J Mol Biol 299: 27-51.

Kaper, J.B., Nataro, J.P., and Mobley, H.L. (2004) Pathogenic Escherichia coli. Nat Rev Microbiol 2: 123-140.

Kennelly, P.J., and Potts, M. (1996) Fancy meeting you here! A fresh look at 'prokaryotic' protein phosphorylation. J Bacteriol 178: 4759-4764.

Krell, T., Lacal, J., Busch, A., Silva-Jimenez, H., Guazzaroni, M.E., and Ramos, J.L. (2010) Bacterial sensor kinases: diversity in the recognition of environmental signals. Annu Rev Microbiol 64: 539-559.

Leonard, C.J., Aravind, L., and Koonin, E.V. (1998) Novel families of putative protein kinases in bacteria and archaea: evolution of the 'eukaryotic' protein kinase superfamily. Genome Res 8: 1038-1047.

Livny, J., and Friedman, D.I. (2004) Characterizing spontaneous induction of Stx encoding phages using a selectable reporter system. Mol Microbiol 51: 1691-1704.

Mascher, T., Helmann, J.D., and Unden, G. (2006) Stimulus 
perception in bacterial signal-transducing histidine kinases. Microbiol Mol Biol Rev 70: 910-938.

Matsushiro, A. (1961) Isolation of UV-inducible temperate phage phi80. Biken J 4: 133-135.

Matz, K., Schmandt, M., and Gussin, G.N. (1982) The rex gene of bacteriophage lambda is really two genes. Genetics 102: 319-327.

O'Brien, A.D., Newland, J.W., Miller, S.F., Holmes, R.K., Smith, H.W., and Formal, S.B. (1984) Shiga-like toxinconverting phages from Escherichia coli strains that cause hemorrhagic colitis or infantile diarrhea. Science 226: 694696.

Oppenheim, A.B., and Court, D. (1983) Phage lambda's accessory genes. In Lambda II. Hendrix, R.W., Roberts, J.W., Stahl, F.W., and Weisberg, R.A. (eds). Cold Spring Harbor, NY: Cold Spring Harbor Press, pp. 251277.

Parma, D.H., Snyder, M., Sobolevski, S., Nawroz, M., Brody, E., and Gold, L. (1992) The Rex system of bacteriophage lambda: tolerance and altruistic cell death. Genes Dev 6: 497-510.

Pereira, S.F., Goss, L., and Dworkin, J. (2011) Eukaryote-like serine/threonine kinases and phosphatases in bacteria. Microbiol Mol Biol Rev 75: 192-212.

Pike, A.C., Rellos, P., Niesen, F.H., Turnbull, A., Oliver, A.W., Parker, S.A., et al. (2008) Activation segment dimerization: a mechanism for kinase autophosphorylation of nonconsensus sites. EMBO J 27: 704-714.

Plunkett, G., 3rd, Rose, D.J., Durfee, T.J., and Blattner, F.R. (1999) Sequence of Shiga toxin 2 phage 933W from Escherichia coli O157:H7: Shiga toxin as a phage lategene product. J Bacteriol 181: 1767-1778.

Ptashne, M., Backman, K., Humayun, M.Z., Jeffrey, A., Maurer, R., Meyer, B., and Sauer, R.T. (1976) Autoregulation and function of a repressor in bacteriophage lambda. Science 194: 156-161.

Reichardt, L., and Kaiser, A.D. (1971) Control of lambda repressor synthesis. Proc Natl Acad Sci USA 68: 21852189.

Robert, J., Sloan, S.B., Weisberg, R.A., Gottesman, M.E., Robledo, R., and Harbrecht, D. (1987) The remarkable specificity of a new transcription termination factor suggests that the mechanisms of termination and antitermination are similar. Cell 51: 483-492.

Robertson, E.S., Aggison, L.A., and Nicholson, A.W. (1994) Phosphorylation of elongation factor $G$ and ribosomal protein S6 in bacteriophage T7-infected Escherichia coli. Mol Microbiol 11: 1045-1057.

Roskoski, R., Jr (2004) Src protein-tyrosine kinase structure and regulation. Biochem Biophys Res Commun 324: 1155-1164.

Sambrook, J., Fritsch, E., and Maniatis, T. (1989) Molecular Cloning, A Laboratory Manual. Cold Spring Harbor, NY: Cold Spring Harbor Laboratory Press.

Schumacher, M.A., Piro, K.M., Xu, W., Hansen, S., Lewis, K., and Brennan, R.G. (2009) Molecular mechanisms of HipAmediated multidrug tolerance and its neutralization by HipB. Science 323: 396-401.

Scotland, S.M., Smith, H.R., Willshaw, G.A., and Rowe, B.
(1983) Vero cytotoxin production in strain of Escherichia coli is determined by genes carried on bacteriophage. Lancet 2: 216.

Shatzman, A., Ho, Y.-S., and Rosenberg, M. (1983) Use of phage lambda signals to obtain efficient expression of genes in Escherichia Coli. In Experimental Manipulation of Gene Expression. Inouye, M. (ed.). Orlando: Academic Press Inc, pp. 1-14.

Shub, D.A. (1994) Bacterial viruses. Bacterial altruism? Curr Biol 4: 555-556.

Smith, J.A., Francis, S.H., and Corbin, J.D. (1993) Autophosphorylation: a salient feature of protein kinases. Mol Cell Biochem 127-128: 51-70.

Snyder, L. (1995) Phage-exclusion enzymes: a bonanza of biochemical and cell biology reagents? Mol Microbiol 15: 415-420.

Sussman, R., and Jacob, F. (1962) Sur un systeme de repression thermosensible chez le bacteriophage lambda d'Escherichia coli. C R Acad Sci Paris 254: 1517-1519.

Tyler, J.S., and Friedman, D.I. (2004) Characterization of a eukaryotic-like tyrosine protein kinase expressed by the Shiga toxin-encoding bacteriophage 933W. J Bacteriol 186: 3472-3479.

Tyler, J.S., Mills, M.J., and Friedman, D.I. (2004) The operator and early promoter region of the Shiga toxin type 2-encoding bacteriophage $933 \mathrm{~W}$ and control of toxin expression. J Bacteriol 186: 7670-7679.

Tyler, J.S., Livny, J., and Friedman, D.I. (2005) Lambdoid phages and Shiga toxin. In Phage: Role in Pathogenesis and Biotechnology. Waldor, M.K., Friedman, D.I., and Adhya, S. (eds). Washington, DC: ASM Press, pp. 131164.

Van Melderen, L., and Saavedra De Bast, M. (2009) Bacterial toxin-antitoxin systems: more than selfish entities? PLOS Genet 5: e1000437.

West, S.A., and Gardner, A. (2010) Altruism, spite, and greenbeards. Science 327: 1341-1344.

Wulff, D.L., and Rosenberg, M. (1983) Establishment of repressor synthesis. In Lambda II. Hendrix, R.W., Roberts, J.W., Stahl, F.W., and Weisberg, R.A. (eds). Cold Spring Harbor, NY: Cold Spring Harbor Laboratory, pp. 53-73.

Yamamoto, N., Ushijima, N., Gemski, P., and Baron, L.S. (1977) Genetic studies of hybrids between coliphage lambda and salmonella phage P22: genetic analysis of the P22-lambda hybrid class. Mol Gen Genet 155: 117-121.

Yamamoto, N., Wohlhieter, J.A., Gemski, P., and Baron, L.S. (1978) lambdaimm P22dis: a hybrid of coliphage lambda with both immunity regions of Salmonella phage P22. Mol Gen Genet 166: 233-243.

Yarmolinsky, M.B. (1971) Making and Joining DNA Ends. In The Bacteriophage Lambda. Hershey, A.D. (ed.). Cold Spring Harbor, NY: Cold Spring Harbor Laboratory, pp. 97-109.

Yarmolinsky, M.B. (1995) Programmed cell death in bacterial populations. Science 267: 836-837.

Yu, D., Ellis, H.M., Lee, E.C., Jenkins, N.A., Copeland, N.G., and Court, D.L. (2000) An efficient recombination system for chromosome engineering in Escherichia coli. Proc Natl Acad Sci USA 97: 5978-5983. 